\title{
Planificación e implementación parcial del sistema de gestión de la calidad en la empresa diseños Punto de Oro Ltda.
}

Charry Velásquez Hialmar, Duque Beltrán Paola Andrea, Rui Rodrígue: Andrea ${ }^{l}$

\section{Resumen}

Diseños Punto de Oro Ltda. es una compañía privada dedicada a la fabricación y comercialización de corbatas; La empresa inició la planificación e implementación parcial del sistema de gestión de la calidad (procesos de realización de producto), de acuerdo con los requisitos de la NTC - ISO 9001 de 2000. Como problema a resolver se definió la permanente variación en la calidad de las corbatas entre cada lote de producción. Durante la ejecución del proyecto, como es común en este tipo de trabajos, se presentaron obstáculos para la obtención de los resultados, los cuales fueron superados por el equipo de trabajo y se obtu-

1 Charry Velasquez Hialmar, Ingeniero Civil, Especialista en Administración y Gerencia de Sistemas de la Calidad.

Duque Beltran Paola Andrea, Bacterióloga, Especialista en Administración y Gerencia de Sistemas de la Calidad.

Ruiz Rodríguez Andrea, Ingeniera Industrial, Especialista en Administración y Gerencia de Sistemas de la Calidad. vieron grandes aprendizajes que se comparten en el presente artículo.

Palabras clave

Calidad de las corbatas, implementación, Sistema de Gestión de la Calidad, variación.

\section{Abstract}

Diseños Punto de Oro Ltda. is a private company in the production and commercialization of ties; the company began the planning and partial implementation of the quality managment system, in accordance with ISO 9001:2000. The frequent variation in the quality of ties between each batch of production was the problem to solve. There was obstacles in the project, wich was overcome by the team and were great learning to be shared in this article.

Keywords

Quality of the ties, implementation, System of Administration of the Quality, variation. 


\section{INTRODUCCIÓN}

El presente artículo describe el proceso de desarrollo del proyecto: Planificación e implementación parcial del sistema de gestión de la calidad en la empresa Diseños Punto de Oro Ltda.

Diseños Punto de Oro cuenta actualmente con 19 empleados en su planta de personal. La compañía tiene dos líneas de producto, las cuales son: corbata de dotación (para empresas y colegios) fabricadas en telas nacionales y corbata de colección (para almacenes de ropa principalmente) fabricadas con telas importadas. Como objetivo del proyecto se definió: planificar e implementar parcialmente (procesos de realización de producto) el Sistema de Gestión de la Calidad. En cuanto al problema central definido, es necesario aclarar que cada lote equivale a 20 corbatas y la variación de calidad se refiere a las medidas del producto final, acabados y material y se presenta en un $15 \%$ de cada lote, lo cual equivale a 3 unidades.

Para el desarrollo del proyecto fueron de suma importancia las metodologías empleadas para la ejecución de las actividades planeadas, así como el seguimiento a las mismas. El proyecto llevó a formular conclusiones respecto a los procesos de planificación e implementación de los sistemas de gestión de calidad en las microempresas colombianas y por lo tanto al aprendizaje de una serie de factores que es importante tener en cuenta en el momento de emprender este tipo de proyectos.

A través de la implementación se busca dar solución permanente al problema anteriormente nombrado, obteniendo beneficios a mediano y largo plazo tales como la disminución de quejas, reclamos y devoluciones por mala calidad, y esto a su vez contribuye a mejorar la rentabilidad, sostenibilidad y crecimiento de la organización; así como al incremento de la satisfacción del cliente por el cumplimiento de sus requisitos frente al producto ofrecido por la empresa. Además, no se debe dejar de lado la importancia que tiene desde hace unos años para las organizaciones establecer, documentar e implementar un sistema de gestión de la calidad para incrementar su competitividad y productividad en el mercado.

Además de los anteriores beneficios, el equipo del proyecto se planteó de generar conocimiento para las microempresas que desean comenzar el camino de la implementación de un sistema de gestión de la calidad y mostrar los beneficios que pueden obtener si se hacen las cosas bien desde el principio.

\section{Formulación de objetivos}

\section{Objetivo general}

Planificar e implementar parcialmente (procesos de realización de producto) el Sistema de Gestión de la Calidad para la Empresa Diseños Punto de Oro Ltda.

\section{Objetivos específicos}

- Realizar un análisis de la variación presentada entre cada lote de producción.

- Concientizar al personal de la organización sobre la importancia de implementar el Sistema de Gestión de la Calidad en la organización.

- Proponer el funcionamiento por procesos en la organización y dar las pautas necesarias para llevarlo a cabo.

- Comprometer a la alta dirección de la organización con la implementación del Sistema.

- Elaborar el Manual de la Calidad en cuanto a los procesos definidos dentro del alcance del proyecto.

- Realizar prueba piloto para evaluar la disminución de la variación. 


\section{METODOLOGÍA}

Para el desarrollo de nuestra investigación tuvimos en cuenta los siguientes aspectos metodológicos, que nos llevaron al logro de los objetivos:

1. Diagnóstico: consulta de documentación proporcionada por la empresa en medio físico y en medio magnético. Para lo anterior se recurrió a fuentes tales como: reportes e informes de producción, estadísticas y estudios de mercadeo, bases de datos de la empresa, percepción de empleados, observación directa a los procesos ejecutados con el fin de concluir y generar un balance inicial.

2. Medición de tiempos: realizada en el proceso de producción con el fin de identificar cuellos de botella y fuentes de la permanente variación de la calidad del producto terminado entre cada lote de producción. Para la medición se realizaron tres muestras para contar con datos confiables, posteriormente se calculó un tiempo parcial de cada operación y el tiempo estándar total del proceso de fabricación de una unidad.

3. Reuniones de trabajo: realización de charlas formales e informales con la alta dirección de la empresa y con otros empleados cuando fue necesario. Las operarias jugaron un papel clave en la información levantada durante el proyecto, ya que estas empleadas son las que ejecutan las operaciones en las cuales se puede causar la variación entre cada lote de producción.

4. Entrevistas formales: con el fin de levantar la información relativa a la documentación necesaria para llevar a cabo el proyecto. Las llamamos formales ya que en ellas se dejó evidencia de lo realizado por medio de grabaciones y actas documentadas.
5. Encuestas: a clientes de la organización con el fin de identificar sus requisitos. Las encuestas fueron realizadas a usuarios finales de las corbatas.

6. Presentaciones: intervenciones realizadas ante la organización con el fin de sensibilizar y capacitar frente a los sistemas de gestión de calidad, además de presentar los avances del proyecto.

7. Asignación de responsabilidades: se refiere a asignación de responsabilidades a la organización tales como: revisiones a los avances del proyecto, compromiso con su ejecución, suministro de información, etc. De igual forma, distribución de tareas dentro del equipo de trabajo para llevar a cabo el plan de trabajo.

\section{ANÁLISIS}

De acuerdo con los datos recogidos en el proyecto a través de las metodologías anteriormente nombradas surgió la siguiente información:

- En la operación de forrado, dentro del proceso de producción, es donde se presenta el mayor número de variaciones en cada lote de producción.

- La operaria de forrado influye en el resultado de la operación, a pesar de que ésta es realizada con una máquina, debido a que la distancia que se deja entre el extremo de la tela y el forro se mide visualmente, sin usar ningún equipo de medición.

- Existen 8 procesos implementados por la empresa al iniciar el proyecto, pero ninguno se encuentra documentado, así como tampoco se cuenta con instructivos, guías o manuales que documenten el método para llevar a cabo las actividades propias del proceso de producción 
- No existen registros de no conformidades, causas de las mismas, estadísticas, etc., así como tampoco se lleva a cabo evaluación de proveedores, ni control de documentos y registros que permitan identificar otros aspectos para la mejora.

- No existe planificación de los procesos y éstos son ejecutados empíricamente y sin metas claras para su gestión.

- Relacionado con lo anterior se evidenció que no existen mecanismos de medición de los procesos que permitan realizar seguimiento al desempeño de los mismos.

\section{RESULTADOS}

\section{Para la empresa Diseños Punto de Oro se obtuvieron los siguientes resultados:}

Con la ejecución del proyecto se logró sensibilizar a la alta dirección respecto a los beneficios obtenidos con la implementación de un sistema de gestión de calidad, a la importancia de planificar las actividades diarias y de contar con registros que permitan realizar análisis y tomar decisiones basadas en hechos y datos confiables. Además se hizo entrega de la política, objetivos de calidad, mapa de procesos (ver figura 1) y documentación de los mismos, lo cual permitirá a la empresa continuar con la implementación de su sistema.

El impacto más importante a resaltar es la concientización de la empresa con los beneficios de iniciar un proceso formal de implementación del sistema y su manifestación de querer realizar este proceso, no sólo con el fin de conseguir una certificación de calidad, sino para mejorar en su gestión, resolver sus problemas y lograr sus objetivos.

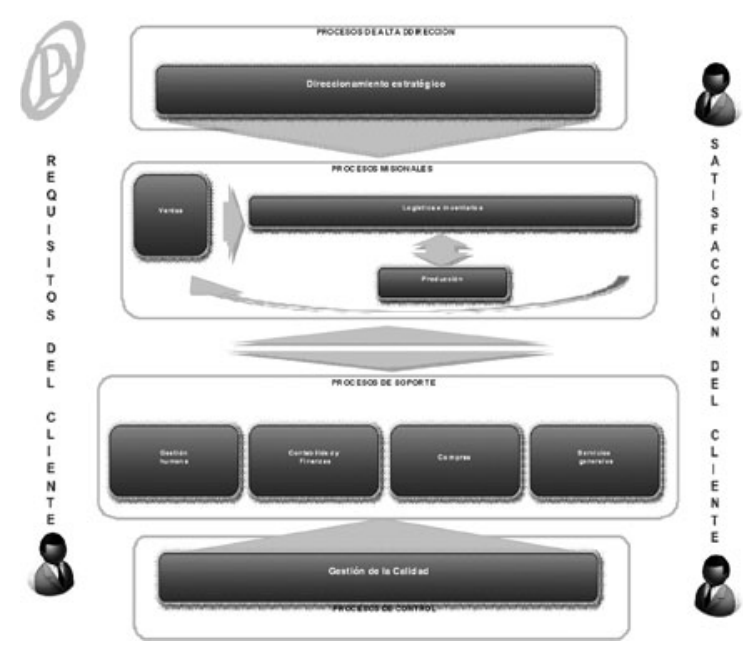

Figoura 1. Mapa de procesos

\section{Para los investigadores se obtuvieron los siǵuientes resultados:}

Los resultados como grupo de investigación están directamente relacionados al aprendizaje obtenido durante su desarrollo, en el cual se destaca la importancia que tienen los planes, programas y cronogramas en un proyecto, cuyo correcto uso se convierte en una herramienta fundamental para el logro de los resultados. Además se pueden resaltar otros resultados positivos, detallados en el numeral VII (Lecciones aprendidas).

\section{CONCLUSIONES}

- La variación de la calidad del producto es solucionable en la medida en que la alta dirección se ponga al frente de la implementación de las recomendaciones realizadas por el equipo del proyecto.

- El compromiso de la alta dirección frente a la implementación es fundamental para el éxito de la misma, sin embargo, es importante sensibilizar y concientizar a todo el personal involucrado en la empresa, ya que éste es el responsable de ejecutar los procesos y por lo tanto puede identificar oportunidades de mejora continuamente. 
- Las microempresas de tipo familiar tienen un gran reto cuando deciden emprender la implementación de un sistema de gestión de calidad debido a sus dificultades técnicas, económicas y de gestión a las que se enfrentan a diario, por lo tanto es conveniente que este proceso sea planeado por etapas y de acuerdo con las posibilidades de la organización, sin ser demasiado ambiciosos en el planteamiento de objetivos y metas.

- Aunque el problema del cual se partió es de importancia y debe resolverse, la empresa cuenta con otros inconvenientes que deben analizarse y buscar sus soluciones, ya que de ellos parten muchas de las dificultades por las cuales atraviesa la organización, como por ejemplo la falta de planificación a nivel general, deficiencias en las actividades de mercadeo realizadas, falta de comunicación entre los empleados y la alta dirección, etc.

- La generación y el control de los registros en organizaciones de este tipo son de suma importancia y deben hacerse esfuerzos para que sean tenidos en cuenta en la toma de decisiones, ya que muchas de ellas son tomadas por la urgencia pero sin previo análisis. Lo anterior se hace más importante si se tiene en cuenta que la persona que está al frente de la empresa realiza una administración empírica.

\section{RECOMENDACIONES}

Respecto al problema tratado en el proyecto se sugieren las siguientes recomendaciones:

- Nombrar a un empleado fijo que ejecute las funciones de Jefe de Planta.

- Implementar controles en el proceso de producción y generar registros de éstos.
- Realizar una verificación directa de la ejecución de estos controles.

- Registrar las no conformidades y sus causas con el fin de generar acciones correctivas.

- Es importante que se realicen análisis de causas de las desviaciones del producto terminado, en conjunto entre la alta dirección y las operarias.

- Evaluar a los proveedores de materias primas con el fin de asegurar la calidad de los materiales comprados.

- Emplear los resultados de estas evaluaciones para tomar decisiones de continuar o no con dichos proveedores.

- Establecer acuerdos de servicio principalmente con los proveedores extranjeros, ya que actualmente existe materia prima obsoleta y en malas condiciones de almacenamiento, lo que ocasiona la fabricación de productos defectuosos Lo anterior se presenta, según información proporcionada por la alta dirección, debido al alto costo de la devolución de los materiales hasta su país de origen y el no reconocimiento (económico) de los defectos por parte de los proveedores.

- Implementar acciones como el uso de guías en las máquinas para la medición del forrado, unión de piezas y marquillado.

- Realizar capacitación al personal que labora actualmente en la planta para que emplee las guías implementadas.

- Realizar seguimiento a la implementación de las acciones con el fin de asegurarse de su eficacia.

- Realizar inducción al cargo a todo el personal de producción que ingrese a la empresa. Se propone 
entregar una copia del instructivo para confección de corbatas elaborado por el equipo de trabajo.

- Realizar control de calidad al finalizar el proceso de acuerdo con parámetros de control propuestos. Se propone realizar un check list con los aspectos que deben tenerse en cuenta en este control (por ejemplo mediciones, acabados, postura de marquillas, etc.).

- Respecto al instrumento de medición (metro de modistería), se propone adoptar las siguientes prácticas:

- Todos los metros utilizados en el proceso de confección de la corbata deben ser de la misma marca.

- Todos los metros utilizados en el proceso de confección se deben reemplazar obligatoriamente cada seis meses o antes, cuando alguno de ellos presente algún tipo de deterioro o daño durante la manipulación que afecte el proceso de medición.

- Establecer planes de capacitación para las personas involucradas en la fabricación del producto de acuerdo con los recursos de que dispone la empresa.

Respecto al desarrollo del proyecto se sugiere:

- Continuar con la implementación del sistema, para lo cual se debería realizar talleres prácticos, reuniones con la alta dirección, llevar a cabo charlas sobre la importancia del tema y sobretodo que la persona delegada como representante de la alta dirección sea capacitado en cuanto al tema de la calidad.

- Capacitar al personal en las actividades que ejecutan.

- Planificar los procesos ejecutados en la organización.
- Contar con una persona que se haga responsable de la gestión de la calidad dentro de la organización y lleve a cabo las actividades necesarias para ello.

\section{LECCIONES APRENDIDAS}

Para el equipo de trabajo el desarrollo de este proyecto fue de gran importancia y dejó una serie de aprendizajes que a continuación compartimos con los lectores con el fin de que sean tenidos en cuenta en la ejecución de proyectos académicos relacionados.

- Para la realización de la implementación del sistema de gestión de la calidad se deben llevar a cabo las actividades planeadas de acuerdo con el cronograma definido inicialmente y procurar trabajar con tiempos de holgura para evitar retrasos.

- El conocimiento total de la empresa del equipo involucrado es fundamental, ya que esto hace más objetivas, prácticas y reales sus apreciaciones y recomendaciones.

- Los grupos o personas más impactados directamente en sus actividades con la implementación de un sistema de gestión de calidad, tales como empleados operativos de las microempresas de este tipo, son actores capaces de impulsar iniciativas que contribuyan al mejoramiento de la gestión empresarial, por lo anterior, deben ser considerados a la hora de diseñar y ejecutar estos proyectos. Esto facilita la generación de ideas y la búsqueda de la raíz de los problemas.

- La necesidad de compatibilizar las necesidades de la organización que emprende el proyecto y las demás personas que intervienen en él. Aunque siendo imprescindible su aporte, los profesionales expertos que participan en estos proyectos, en ciertas ocasiones, por tener un interés más bien acadé- 
mico, no son del todo sensibles a las problemáticas prácticas y reales de la empresa.

- Se debe mantener un equilibrio en la conformación de los equipos que deben intervenir en el proyecto. Esto quiere decir que sean tenidos en cuenta personal estratégico, operativo y profesionales expertos con el fin de lograr opiniones y acciones mucho más transversales a los procesos de la organización.

- Una forma de superar la desconfianza en los beneficios de la implementación de un sistema de gestión de calidad por parte de la alta dirección de las microempresas de tipo familiar es integrar a profesionales que destaquen las victorias o avances tempranos, teniendo en cuenta que este tipo de organizaciones requieren de resultados medibles a corto tiempo por sus dificultades de tipo económico.

- Otra forma de superar la desconfianza antes mencionada es crear mecanismos de medición y evaluación de todas las partes involucradas en el proyecto respecto a la marcha de los objetivos del proyecto. Esto puede ser llevado a cabo por auditorías internas.

- Es conveniente usar y divulgar casos reales exitosos de implementación en otras empresas del mismo tipo y así promover la capitalización de experiencia y conocimientos adquiridos por todos los participantes en el proyecto.

- Es necesario considerar en el diseño y gestión de estos proyectos, desde su inicio, el contexto cultural de la organización, con el fin de no indisponer o crear sensaciones de que se busca cambiar todo lo llevado a cabo por la empresa hasta el inicio del proyecto porque nada funciona bien.
- El equipo de profesionales responsable del proyecto debe trabajar como piezas muy bien engranadas, sin nunca caer en duplicidad de tareas u opiniones contradictorias que puedan generar desconfianza a la organización.

- Un aspecto negativo que se resalta es la falta de información frente a los beneficios de la ejecución de un proyecto como éste, tales como: mejora en la ejecución de los procesos, incremento de la satisfacción del cliente, disminución de costos, mejor aprovechamiento de los recursos, etc., en algunos sectores de la economía, ya que sólo se le da relevancia al certificado de calidad sin tener en cuenta que no es un fin sino un medio para la mejora.

- El quipo de profesionales del proyecto en ocasiones le da mucha relevancia a la forma de presentación o utilización de equipos tecnológicos que impresionen a la alta dirección de la organización en la presentación de avances.

- Lo importante de ello, es que los avances, informes, intervenciones y demás sean de fácil entendimiento para todo el personal de la organización, sin importar el medio, ya que no se debe olvidar que no todos los involucrados son expertos y el equipo debe ser conductor de aprendizaje en la implementación del proyecto. Por lo tanto, como aprendizaje respecto a la ejecución del presupuesto, los rubros deben ir más enfocados al trabajo profesional y no al gasto en equipos, impresiones de alta calidad 0 este tipo de temas. 


\section{Referencias}

Conjunto de documentos para la introducción y el soporte de la serie de Normas ISO 9000. Orientación sobre el concepto y el uso del "Enfoque basado en procesos" para los sistemas de gestión, pp. 3-4.

Linares Vélez, Guillermo y Perdomo Medina, Manuel. Cómo hacer procedimientos. Segunda actualización. Bogotá: Filigrana, 2002.
Pope, Jefrey. Investigación de mercados. New York: Normos, $6^{a}$. Ed., 1992.

Instituto Colombiano de Normas Técnicas y CertiFICACIÓN, ICONTEC. Norma Técnica Colombiana NTC-ISO 9001: 2000. Bogotá, 2006. 